\title{
The perception of size and distance under monocular observation
}

\author{
ATSUKI HIGASHIYAMA \\ University of Osaka Prefecture, Osaka 591, Japan
}

\begin{abstract}
A relative-perceived-size hypothesis is proposed to account for the perception of size and distance under monocular observation in reduced-cue settings. This hypothesis is based on two assumptions. In primary processing, perceived size is determined by both proximal stimulation on the retina and distance information from primary cues such as oculomotor cues. In secondary processing, the relation of two primary perceived sizes determines another relation of secondary perceived distances, so that an object of smaller primary perceived size is judged to be further away. An experiment was designed to test this hypothesis, especially the assumption of secondary processing, by making ratio judgments of perceived size and perceived distance for two successively presented targets. The standard square was presented at a constant distance and varied in visual angle; the variable square was presented with a constant visual angle in distance. The results showed that an inverse relation between size and distance estimates held regardless of whether the visual angles of the targets were the same or different.
\end{abstract}

When two objects of similar shape but different retinal image size are presented successively or simultaneously under reduced-cue conditions, the object of smaller image size is judged to be further away than the object of larger image size. This phenomenon has been observed in a number of studies (Epstein \& Landauer, 1969; Gogel, 1969; Gogel \& Sturm, 1971), and the variable determining perceived relative distance has been assumed to be relative retinal size. According to this conventional hypothesis, if the visual angles of the objects are equal to one another, the observer will perceive them either at the same distance (mainly Gogel, 1969) or at uncertain distances (Rock \& McDermott, 1964), and if they are different, the observer will perceive the further distance to correspond with the smaller visual angle.

The alternative to the relative-retinal-size hypothesis is the relative-perceived-size hypothesis (Higashiyama, 1977), which rests on the following assumptions. The first assumption is that primary distance cues operate even under completely reduced-cue conditions and provide registered distance, an assumption supported by a number of studies (Leibowitz \& Moore, 1966; Leibowitz \& Owens, 1975; Leibowitz, Shiina, \& Hennessey, 1972; Owens \& Leibowitz, 1976). The perceptual system then transforms retinal image size into perceived size by taking into account the registered distance resulting from primary distance cues. We shall refer to this as the primary process. In primary processing, size perception presupposes distance per-

Requests for reprints should be addressed to Atsuki Higashiyama, Department of Psychology, University of Osaka Prefecture, Mozu-umemachi, Sakai City, Osaka 591, Japan. ception. The second assumption is that a ratio of two primary perceived sizes determines a ratio of secondary perceived distances, which differ from primary registered distances produced by primary distance cues. The object of smaller primary perceived size is judged to be further away than that of larger primary perceived size. We shall refer to this as the secondary process. In secondary processing, size perception precedes distance perception.

Generally speaking, primary processing uses any of the classical distance cues to provide as accurate as possible an estimate of distance for use in constructing perceived size. Primary distance cues can be divided into two subgroups-proprioceptive or oculomotor cues (e.g., accommodation and convergence), and visual cues (e.g., binocular retinal disparity and motion parallax). The relative-perceived-size hypothesis is perhaps best applied to the reduced-cue situation where acommodation and accommodative vergence are available, because, in this situation, the perceptual system easily ignores the primary registered distance and accepts the secondary perceived distance (cf. Biersdorf, 1966; Heinemann, Tulving, \& Nachmias, 1959; Ono, Muter, \& Mitson, 1974). On the other hand, if primary processing uses binocular retinal disparity or motion parallax as primary distance cues, then the perceptual system may fail to produce the secondary perceived distance as an overt response, because these visual cues provide the primary perceived distance in a more stable and compelling way than do oculomotor cues.

Contrary to the relative-retinal-size hypothesis, the present hypothesis implies that the relative size cue to distance is defined in terms of relative perceived size. Therefore, even if the visual angles of two 
objects are equal, as long as they do not appear to be equal in perceived size, the observer will not experience them as equidistant or at uncertain distances.

The relative-perceived-size hypothesis suggests the occurrence of the size-distance paradox-the finding that, contrary to the size-distance invariance hypothesis, perceived size and perceived distance are inversely rather than directly related for a constant visual angle in reduced-cue situations. Heinemann et al. (1959) presented in quick succession two stimulus objects that subtended a constant visual angle, but with the standard closer than the variable. However, most observers, viewing monocularly, judged the standard to be further away than the variable. Biersdorf (1966) confirmed these results under a similar viewing condition. Both Grant (1942) and Hermans (1954) reported paradoxical distance judgments for some observers even under binocular observations.

The purpose of this study was (1) to determine whether the relative-retinal-size hypothesis or the relative-perceived-size hypothesis provides a better account of distance perception under a reduced-cue condition where primary distance cues are limited to accommodation and accommodative vergence, and (2) to obtain the necessary data in a systematic way in order to establish quantitatively the paradoxical size-distance relationship.

Ratio judgments of both perceived size and perceived distance were obtained for two squares presented successively at different distances. The comparison target was presented at any one of three different distances so as to subtend the same visual angle. The standard target subtended any one of four visual angles at a constant distance.

Consider judgments of a given standard compared with the three comparison stimuli, with fixed visual angle, but at different locations. According to the relative-retinal-size hypothesis, the changing distance of the comparison should make no difference to either perceived size or perceived distance, since retinal image size does not change: Subjects should judge both relative size and distance to be the same for all comparisons. According to the relative-perceived-size hypothesis, the changing distance of the comparison, registered in primary processing, will combine with fixed retinal image size to make the furthest comparison look largest, the nearest look smallest. Then secondary processing will result in the largest comparison looking nearest, the smallest looking furthest. In short, as distance of comparison increases, the former hypothesis predicts no change in perceived size or distance, whereas the latter predicts that perceived size will increase and perceived distance will decrease.

\section{METHOD}

\section{Observers}

The seven observers were university undergraduates majoring in psychology. They all had normal visual acuity in both eyes.

\section{Apparatus and Stimulus Materials}

A sketch of the mirror arrangement used is shown in Figure 1. A half-silvered mirror (HM) was fixed at a slant of $45^{\circ}$ in the observer's sagittal plane, so that all images of stimulus targets were aligned on the same line of sight for the right eye. The targets were viewed through an aperture $.5 \mathrm{~cm}$ in diameter immediately in front of the eye.

The stimulus targets were square apertures cut in cardboard. Targets $T_{1}, T_{2}$, and $T_{3}$ were the comparison stimuli subtending a visual angle of $1^{\circ} 44^{\prime}$ on a side. They were presented at distances of $33,54.5$, and $133 \mathrm{~cm}$ from the observer's eye position. Targets $\mathrm{T}_{\mathrm{S}}$ were the standard stimuli presented at a distance of $151.5 \mathrm{~cm}$ from the eye, visual angles of which were $2^{\circ} 5^{\prime}, 1^{\circ} 53^{\prime}, 1^{\circ} 44^{\prime}$, and $1^{\circ} 31^{\prime}$ on a side.

The standard and the comparison were presented successively by illuminating them alternately from behind with $20-\mathrm{W}$ bulbs. A frosted glass (FG) was placed in front of each bulb at a distance of $155.5 \mathrm{~cm}$ so as to diffuse the light of the bulb.

\section{Procedure}

The observer was seated on the chair in an observation booth, and his head was positioned in a chinrest so as to align the right eye with the viewing aperture. After a 10-min dark-adaptation period, he viewed the two successively presented targets through the aperture with only the right eye while the left eye remained open. He was then required to assign a value of 10 to the perceived size of the first-presented target and a value of 100 to the perceived distance of the same target. For a given trial, after the shutter had been raised, the first stimulus was illuminated. Ten seconds later, the first stimulus was automatically extinguished and, following a 1-sec interval, the second stimulus was illuminated for $10 \mathrm{sec}$. The observer was required to give the appropriate ratio judgments during the presentation of the second stimulus. When the judgments had been made, the shutter was lowered and the

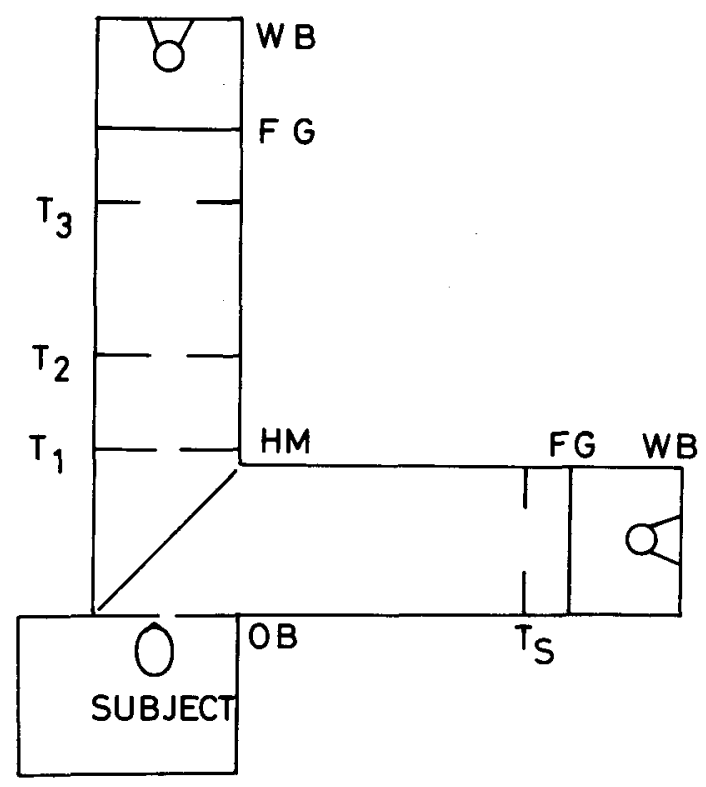

Figure 1. Schematic top-view diagram of the apparatus. 
locations of the stimulus targets were exchanged for the next trial. The experimenter emphasized in the instructions that the observer should not make the judgments until he had attained clear images of the targets in a given trial. If the observer failed to be in focus, he was to signal to the experimenter at once. In this case, the trial was repeated.

The observers received "apparent" instructions. The essential parts of the instructions are: "I am going to present you with a series of squares in total darkness. Two squares will be presented in quick temporal succession. Your task is to tell me how they appear in size and distance by assigning numbers to them. The first square we will call ' 10 ' in size and ' 100 ' in distance, respectively. Your task will be to estimate the apparent size and the apparent distance of the second square. Please try to assign numbers proportional to your subjective impression. I want you to base your judgments on the way that the squares appear. Please disregard any information you may have about the physical or real size and distance of the squares."

Although afterimages might have been produced by gazing at the first target, they did not seem to interfere with the judgments of the second target. The afterimages were weak and no observer confused the targets with the afterimages.

There were 12 possible pairs of stimulus targets, since each of four standard stimuli could occur with each of three comparison stimuli. For each pair, four estimation trials were run, in two of which the standard preceded the comparison, and in the remaining two of which, it followed the comparison. The order of pair presentation was randomly determined for each observer with the restriction that all trials involving any particular standard be performed before the trials involving another standard occurred.

\section{RESULTS AND DISCUSSION}

Each observer's estimates for trials where the standard followed the comparison were transformed so that the perceived size and the perceived distance of the standard square were equal to 10 and 100 , respectively.' Hence, for all trials, values for the comparison that were greater than 10 and 100 meant that it was judged larger and further away, respectively, than the standard. This transformation was possible only if the observers were assumed to make ratio judgments.

Figure 2 shows the results. Each point represents the mean of $28(4 \times 7)$ trials. The parameter is the visual angle of the standard. The left panel of Figure 2 shows the results for size estimates of the comparison plotted against the physical distance of the comparison squares. Note that smaller distances are shown to the right of the abscissa. The reciprocal of the physical distance, which is appropriate to express the relative changes of accommodation in diopter necessary to achieve a clear image, is also indicated on the abscissa. A two-way analysis of variance with repeated measures was performed on the size estimates. In performing this analysis, the means of the four size estimates for each of the 12 pairs of stimulus targets were used as the scores for each observer. The results of this analysis show that the effect of distance of comparison is significant $[\mathrm{F}(2,12)=4.28, \mathrm{p}<.05]$ and the effect of visual angle of standard is significant $[F(3,18)$ $=36.86, \mathrm{p}<.01]$, but the interaction of distance with visual angle is not $(F<1)$.

In a similar way, the right panel of Figure 2 shows the results for the distance estimates of the

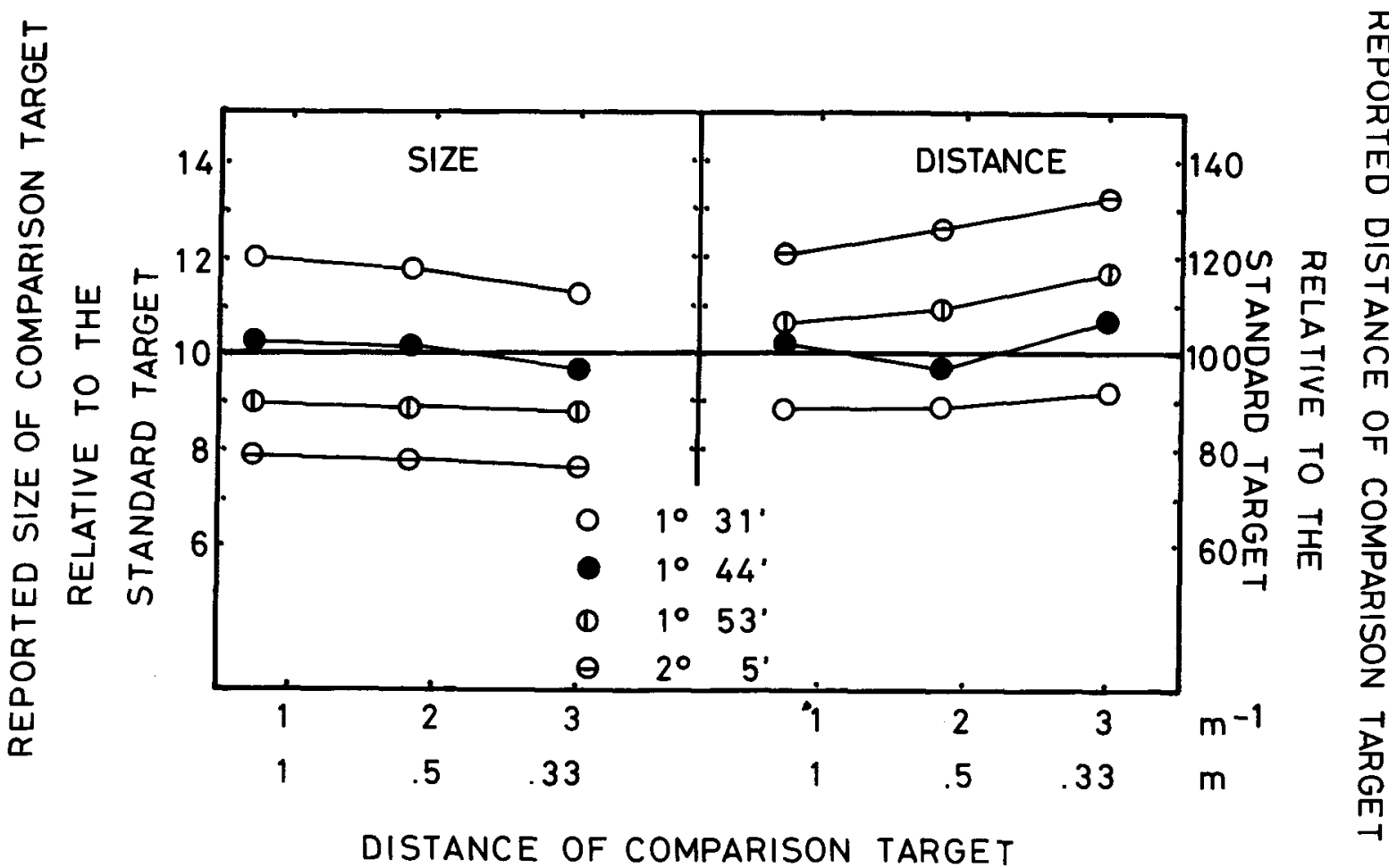

Figure 2. Size estimates and distance estimates of the comparison stimulus plotted against physical distance (m) or the reciprocal of physical distance $\left(\mathrm{m}^{-1}\right)$. The parameter is the visual angle of the standard stimulus. 
comparison. The distance estimates were analyzed by a two-way analysis of variance with repeated measures. The results of this analysis show that the effect of distance of comparison is significant $[\mathrm{F}(2,12)=10.35$, $\mathrm{p}<.01]$, as is the effect of visual angle of standard $[F(3,18)=9.27, p<01]$, while the interaction of distance with visual angle is not $[F(6,36)=1.26$, $\mathrm{p}>$.05].

Thus, the statistical analyses confirm the impressions given by Figure 2: Size estimates of the comparison increase as the visual angle of the standard decreases, and as the physical distance of the comparison increases; distance estimates of the comparison decrease as the visual angle of the standard decreases, and as the physical distance of the comparison increases.

These results do not support the relative-retinal-size hypothesis, which predicts no change in size or distance when a given standard is judged relative to the three comparisons of the same visual angle. They do support the relative-perceived-size hypothesis, which predicts increasing size and decreasing distance judgments as the comparison increases in distance.

For the purpose of clarifying the relation between them, size estimates were plotted against distance estimates in Figure 3. The relation may be reasonably well represented by the equation

$$
S^{\prime}{ }_{C} / S^{\prime} s=D_{S}^{\prime} / D^{\prime} \text {, }
$$

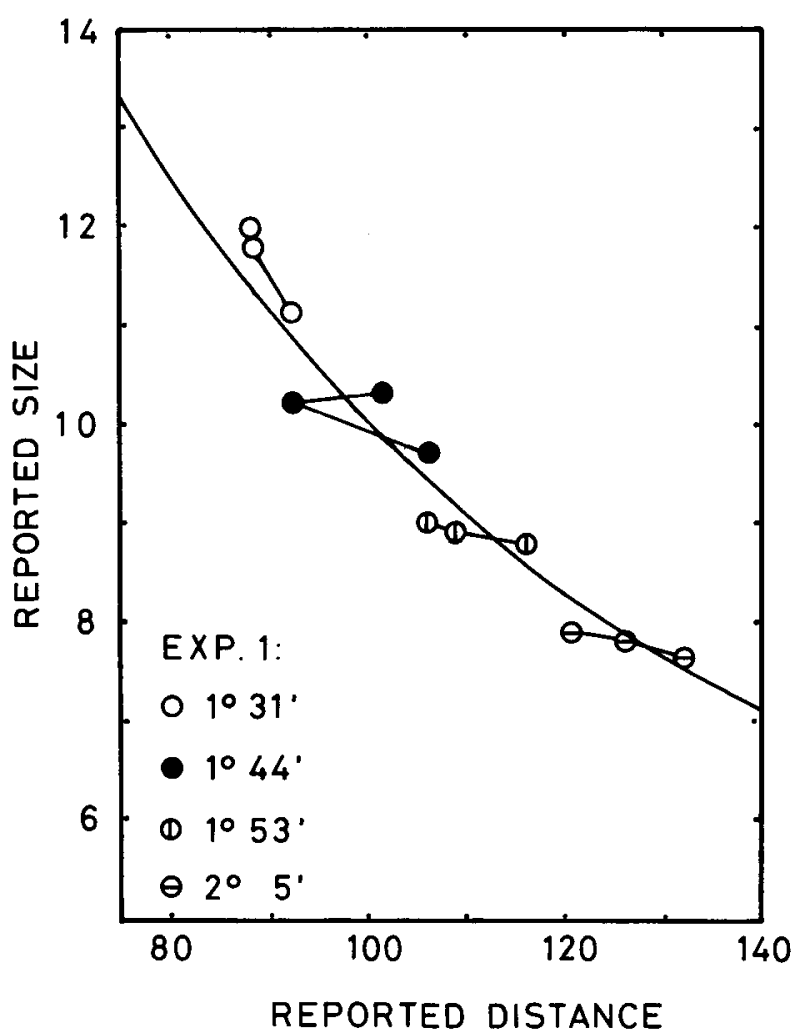

Figure 3. Relation of size estimates and distance estimates. or, more generally,

$$
S^{\prime} \mathrm{C} \cdot \mathrm{D}^{\prime} \mathrm{C}=\mathrm{k} \text {, }
$$

where $S^{\prime} s$ and $D^{\prime} s$ are perceived size and perceived distance of the standard, and $S^{\prime}{ }_{C}$ and $D^{\prime}{ }_{C}$ are those of the comparison. Since, according to Equations 1 and 2 , the value of $\mathrm{k}$ is equal to the product of $S^{\prime} s$ and $D$ 's, that value should be 1,000 in this experiment. This predicted curve is drawn in Figure 3. As simple and convenient measures for direction and degree of discrepancy, the mean and root mean square were computed with respect to the difference between the observed and predicted distance estimates: The mean was .49 and the root mean square was 3.87.

In order to evaluate the prediction that $\mathrm{k}=1,000$, Equation 2 was rewritten as $D^{\prime} \mathrm{C}=k\left(1 / \mathrm{S}^{\prime} \mathrm{C}\right)$, and a least squares line was fitted to the distance estimates as a function of the reciprocal of the size estimates. In making this calculation, the assumption was made that the line should pass through the origin. ${ }^{2}$ The slope is the estimated value of $\mathrm{k}$, which was $1,001.02$, and the correlation between the values of $D^{\prime} C$ and $1 / S^{\prime}{ }_{C}$ was .978 . The mean and root mean square were also obtained to represent the direction and degree of discrepancy: The mean was .39 and the root mean square was 3.86. These results and Figure 3 indicate that the estimated value of $\mathrm{k}$ is broadly comparable with the predicted one.

Another way of evaluating the validity of Equation 2 is to compare the results obtained with similar experimental settings and procedures. Epstein and Landauer (1969, Conditions 2 and 3 ) reported magnitude estimations of the size and distance of a variable relative to a standard when the two stimulus targets had different visual angles. Figure 4 shows the estimated size plotted against the estimated distance. The curve represents the prediction $S^{\prime} C \cdot D^{\prime} C=100$, since the perceived size and distance of the standard were assigned a value of 10 . A systematic discrepancy is seen when the estimated size and distance of the variable were less than those of the standard, but overall the theoretical curve is not a bad fit to the data points.

Gogel and his colleagues seem to distinguish the mechanism underlying the relative size cue to relative perceived distance from the mechanism triggering the size-distance paradox. Gogel (1969) and Gogel and Sturm (1971) defined the relative size cue as a relative retinal size cue. On the other hand, Gogel $(1973,1974)$ assumed that the size-distance paradox tends to occur when an observer perceives an object to be smaller or larger than expected relative to familiar size (in the present experiment, perceived size of the first presented target is assumed to be familiar size). In this sense, the relative perceived distance induced by the relative size cue represents a perceptual event, while the sizedistance paradox contains a cognitive factor. The 


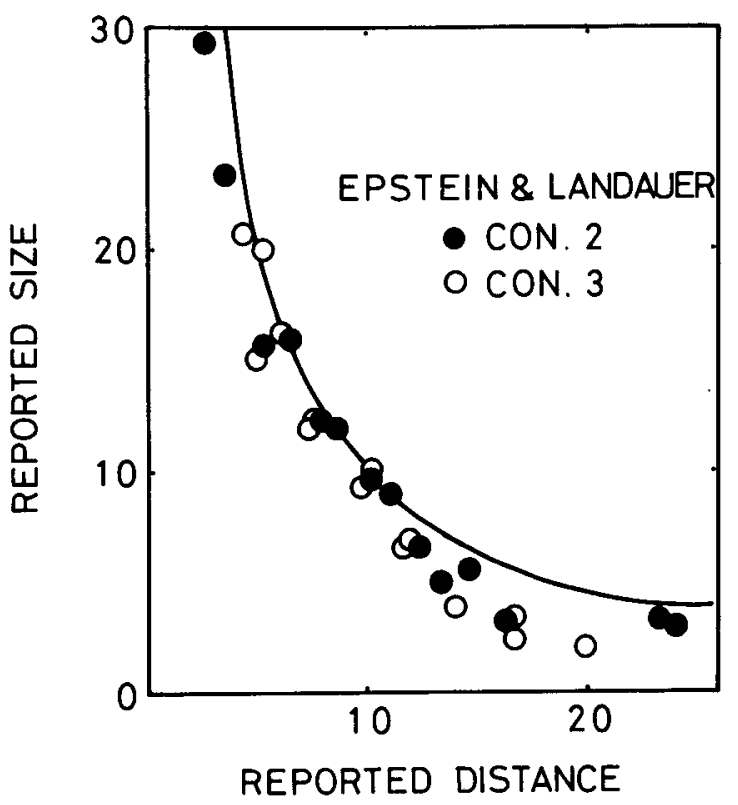

Figure 4. Relation of size estimates and distance estimates from Epstein and Landauer (1969). The solid curve shows the function $S_{C}^{\prime} \cdot D^{\prime}{ }_{C}=100$.

present results may be interpreted by Gogel's hypothesis. However, it seems unparsimonious to assume that the observer's response criteria interchange rapidly, depending on whether the targets are equal to one another in visual size. In contrast, the relativeperceived-size hypothesis may be characterized as an integrative explanation of two seemingly different phenomena.

The relative-perceived-size hypothesis is in a sense far from a new theory. Carlson (1962) and Carlson and Tassone (1962) pointed out that normal adults believe that an object looks smaller at a greater distance. This belief is called the perspective attitude. What is here called the secondary process seems to be in substance equivalent to the perspective attitude. Perhaps, the secondary process, as Carlson has pointed out, is a kind of conceptual coupling between smaller size and further distance or between larger size and closer distance. Rock and Kaufman (1962), in developing a theory of the moon illusion, proposed a mechanism similar to the secondary process to explain the apparent near distance of the apparently larger horizon moon when they supposed that the paradoxical distance judgments depended on the relative size of the horizon and zenith moons.

\section{REFERENCES}

BiERsDorf, W. R. Convergence and apparent distance as correlates of size judgments at near distances. Journal of General Psychology, 1966, 75, 249-264.

Carison, V. R. Size constancy and perceptual compromise. Journal of Experimental Psychology, 1962, 63, 68-73.
Carlson, V. R. \& Tassone, E. P. A verbal measure of perspective attitude. American Journal of Psychology, 1962, 75, 644-647.

Epstein, W., \& Landauer, A. A. Size and distance judgments under reduced conditions of viewing. Perception \& Psychophysics, 1969, 6, 269-272.

GogEL, W. C. The sensing of retinal size. Vision Research, 1969, 9, 1079-1094.

GoGEL, W. C. The organization of perceived space. II. Consequences of perceptual interactions. Psychologische Forschung, 1973, 36, 223-247.

Goget, W. C. Cognitive factors in spatial responses. Psychologia, $1974,17,213-225$.

Gogel, W. C., \& Sturm, R. D. Directional separation and the size cue to distance. Psychologische Forschung, 1971, 35, 57-80.

Grant, V. W. Accommodation and convergence in visual space perception. Journal of Experimental Psychology, 1942, 31, 89-104.

Heinemann, E. G., Tulving, E., \& Nachmias, J. The effect of oculomotor adjustments on apparent size. American Journal of Psychology, 1959, 72, 32-45.

Hermans, T. G. The relationship of convergence and elevation changes to judgments of size. Journal of Experimental Psychology, 1954, 48, 204-208.

Higashiyama, A. Perceived size and distance as a perceptual conflict between two processing modes. Perception \& Psychophysics, 1977, 22, 206-211.

Leibowitz, H., \& Moore, D. Role of accommodation and convergence in the perception of size. Journal of the Optical Society of America, 1966, 56, 1120-1123.

Leibowitz, H. W., \& Owens, D. A. Anomolous myopias and the dark focus of accommodation. Science, 1975, 189, 646-648.

Leibowitz, H. W., Shina, K., \& Hennessy, R. T. Oculomotor adjustments and size constancy. Perception \& Psychophysics, 1972, 12, 497-500.

Ono, H., Muter, P., \& Mitsom, L. Size-distance paradox with accommodative micropsia. Perception \& Psychophysics, 1974, 15, 301-307.

Owens, D. A., \& Leibowitz, H. W. Oculomotor adjustments in darkness and the specific distance tendency. Perception \& Psychophysics, 1976, 20, 2-9.

Rock, I., \& Kaufman, L. The moon illusion, II. Science, 1962, 136, 1023-1031.

Rock, I., \& McDermotT, W. The perception of visual angle. Acta Psychologica, 1964, 22, 119-134.

\section{NOTES}

1. For the trials on which the standard preceded the comparison, no transformation was necessary. For the trials on which the standard followed the comparison, the values of $10^{2}$ and $100^{2}$ were divided by size estimates and distance estimates, respectively.

2. The estimates value of $k$ was calculated from the formula:

$$
\mathbf{k}=\frac{\Sigma \mathrm{D}^{\prime}{ }_{\mathrm{Ci}} / \mathrm{S}_{\mathrm{Ci}}^{\prime}}{\Sigma 1 / \mathrm{S}_{\mathrm{Ci}}^{\prime}} \text {, }
$$

where $\mathrm{i}$ represents any data point shown in Figure 3. This is shown as follows-from the equation: $D^{\prime}{ }_{\mathrm{Ci}}=\mathbf{k}\left(1 / \mathrm{S}^{\prime}{ }_{\mathrm{Ci}}\right)+\varepsilon_{\mathrm{i}}$, we obtain

$$
\Sigma \varepsilon_{i}^{2}=\Sigma\left[D^{\prime}{ }_{C i}-k\left(1 / S^{\prime}{ }_{C i}\right)\right]^{2},
$$

where $\varepsilon_{i}$ is an error that represents the discrepancy between an observed distance estimate and an estimated distance estimate. Taking the derivative of Equation 4 with respect to $k$ and assuming $\mathrm{d} \Sigma \varepsilon_{\mathrm{i}}{ }^{2} / \mathrm{d} \mathbf{k}=0$, we obtain Equation 3 .

(Received for publication June 6, 1978 revision accepted July 20,1979 .) 